\title{
Two Distinct Fluorescent Quantum Clusters of Gold Starting from Metallic Nanoparticles by pH-Dependent Ligand Etching
}

\author{
Madathumpady Abubaker Habeeb Muhammed', Subramani Ramesh', Sudarson Sekhar Sinha², Samir Kumar \\ $\mathrm{Pal}^{2}$, and Thalappil Pradeep ${ }^{1}(\bowtie)$ \\ ${ }^{1}$ DST Unit on Nanoscience (DST UNS), Department of Chemistry and Sophisticated Analytical Instrument Facility, Indian Institute of \\ Technology Madras, Chennai 600 036, India \\ ${ }^{2}$ Unit for Nanoscience and Technology, Department of Chemical, Biological and Macromolecular Sciences, Satyendra Nath Bose National \\ Centre for Basic Sciences, Block JD, Sector III, Salt Lake, Kolkata 700 098, India \\ Received: 30 July 2008/ Revised: 26 August 2008/ Accepted: 27 August 2008 \\ CTsinghua Press and Springer-Verlag 2008. This article is published with open access at Springerlink.com
}

\begin{abstract}
Two fluorescent quantum clusters of gold, namely $\mathrm{Au}_{25}$ and $\mathrm{Au}_{8}$, have been synthesized from mercaptosuccinic acid-protected gold nanoparticles of 4-5 nm core diameter by etching with excess glutathione. While etching at $\mathrm{pH} \sim 3$ yielded $\mathrm{Au}_{25}$, that at $\mathrm{pH} 7-8$ yielded $\mathrm{Au}_{8}$. This is the first report of the synthesis of two quantum clusters starting from a single precursor. This simple method makes it possible to synthesize well-defined clusters in gram quantities. Since these clusters are highly fluorescent and are highly biocompatible due to their low metallic content, they can be used for diagnostic applications.
\end{abstract}

\section{KEYWORDS}

Gold cluster, glutathione, $\mathrm{pH}$, ligand etching, fluorescence

Nanoparticles with a variety of shapes and sizes have been synthesized as they offer numerous possibilities to study size and shape-dependent variations of electronic [1], optical [2], and chemical properties [35]. Quantum clusters are a new class of nanomaterials with only a few atoms in the core and are represented in terms of the number of core atoms such as $\mathrm{Au}_{8}[6$ -9], $\mathrm{Au}_{11}$ [10-14], $\mathrm{Au}_{13}$ [15-17] or $\mathrm{Au}_{25}$ [18-25], rather than their core diameter. Having sub-nanometer size, they cannot possess surface plasmon resonance as the density of states is insufficient to create metallicity. They show discrete energy levels with potentially strong fluorescent transitions between them [26]. Quantum clusters of gold exhibit distinct, but different, fluorescence emissions ranging from ultraviolet to near infrared with increasing number of core atoms [6]. In all the methods reported so far, the clusters are synthesized by reducing the precursor $\mathrm{HAuCl}_{4}$ in presence of templates or protecting species such as dendrimers [6-9], phosphines [1014] and thiols [18-25]. However, these clusters can also be synthesized from metallic nanoparticles using the etching capacity of some ligands which will remove the surface atoms of the metallic nanoparticles leading to stable quantum clusters. Of the various possible clusters synthesized by different methods, some specific clusters are extremely stable compared to others. For example, $\mathrm{Au}_{25} \mathrm{SG}_{18}$, where

Address correspondence to pradeep@iitm.ac.in 
SG is glutathione thiolate, is the most stable cluster among the various glutathione (GSH) protected clusters and the stability of this cluster is attributed to the complete protection of the surface atoms by the ligand [21-24]. The stability of such clusters is due either to the intrinsic stability of the core or the ligand induced stability [19]. Intrinsic stability of cluster cores arises due to closed electronic shells as in $\mathrm{Au}_{8}, \mathrm{Au}_{18}$, etc. or geometric shells as in $\mathrm{Au}_{13}$, $\mathrm{Au}_{55}$, etc. The ligand induced stability arises due to charge transfer, leading to closing of the electronic shell or by full coverage of the surface atoms as in the case of $\mathrm{Au}_{25} \mathrm{SG}_{18}$. Here we report a $\mathrm{pH}$-dependent synthesis of two stable quantum clusters, $\mathrm{Au}_{25}$ and $\mathrm{Au}_{8}$, from mercaptosuccinic acid (MSA) capped gold nanoparticles (Au@MSA) of 4-5 nm core diameter, by ligand etching with glutathione. While etching of $\mathrm{Au} @ \mathrm{MSA}$ with glutathione at $\mathrm{pH} 3$ gives $\mathrm{Au}_{25}$ etching at $\mathrm{pH}$ 7-8 gives $\mathrm{Au}_{8}$. This simple method opens up new possibilities for making an entire range of nanosystems in water, from nanoparticles to quantum clusters, by one-pot solution chemistry. This is the first report of the synthesis of two different clusters from the same metal nanoparticle precursor. This gram-quantity synthetic approach makes it possible to undertake a range of studies on this class of novel materials.

$\mathrm{Au} @ \mathrm{MSA}$ in water was mixed with GSH, 1:4 by weight, in air, under constant stirring at $55{ }^{\circ} \mathrm{C}$. The $\mathrm{pH}$ of the starting solution was in the range 2.73. The UV-vis spectrum was measured at regular intervals during etching. The starting material, Au@ MSA showed a surface plasmon resonance at $540 \mathrm{~nm}$ (Fig. 1), characteristic of metallic nanoparticles. It was found that the surface plasmon resonance at $540 \mathrm{~nm}$ disappeared gradually within a few hours and new absorption features started to appear. After etching twice with glutathione, stable and well-defined peaks similar to $\mathrm{Au}_{25}$ were obtained (see Electronic Supplementary Material (ESM), Fig. S-1) and the surface plasmon resonance disappeared completely. The color of the solution changed from dark brown to reddish brown. A precipitate formed when the mixture was cooled to room temperature, due to the formation of a polymeric gold thiolate. This was centrifuged out and methanol was added to the supernatant to precipitate the clusters. After washing with methanol and ethanol in succession, the sample was freeze dried to afford a dark reddish brown powder. It was redissolved in water and its UV-vis spectrum showed peaks characteristic of $\mathrm{Au}_{25} \mathrm{SG}_{18}$ (Fig. 1) with all the well-structured features and characteristic absorption maximum at $672 \mathrm{~nm}$. Thus it can be concluded that the etching of Au@MSA nanoparticles resulted in the formation of highly water-soluble $\mathrm{Au}_{25}$ clusters. The peak at $672 \mathrm{~nm}(1.55$ $\mathrm{eV})$ arises due to the $\mathrm{LUMO} \leftarrow \mathrm{HOMO}$ transition which can be called an intra-band ( $\mathrm{sp} \leftarrow \mathrm{sp})$ transition $[19,27]$. The features in the lower wavelength region arise due to inter-band transitions $(\mathrm{sp} \leftarrow \mathrm{d})$. The etching was also carried out at $\mathrm{pH}$ 8. In this case, GSH was added to Au@MSA, 1:4 by weight and the $\mathrm{pH}$ was adjusted to 8 by adding $\mathrm{NaOH}$. The color of the solution turned to pale green after 15-20 hours of heating at $60{ }^{\circ} \mathrm{C}$. The solution was centrifuged and the supernatant was precipitated by adding ethanol. After several precipitation and washing cycles, optical absorption was measured (Fig. 1). The surface plasmon resonance had disappeared completely and the optical absorption spectrum matches with that of $\mathrm{Au}_{8}$ synthesized previously [9]; the product obtained was thus assumed to have an $\mathrm{Au}_{8}$ core, pending further characterization (to be described below).

The formation of $\mathrm{Au}_{8}$ was confirmed by

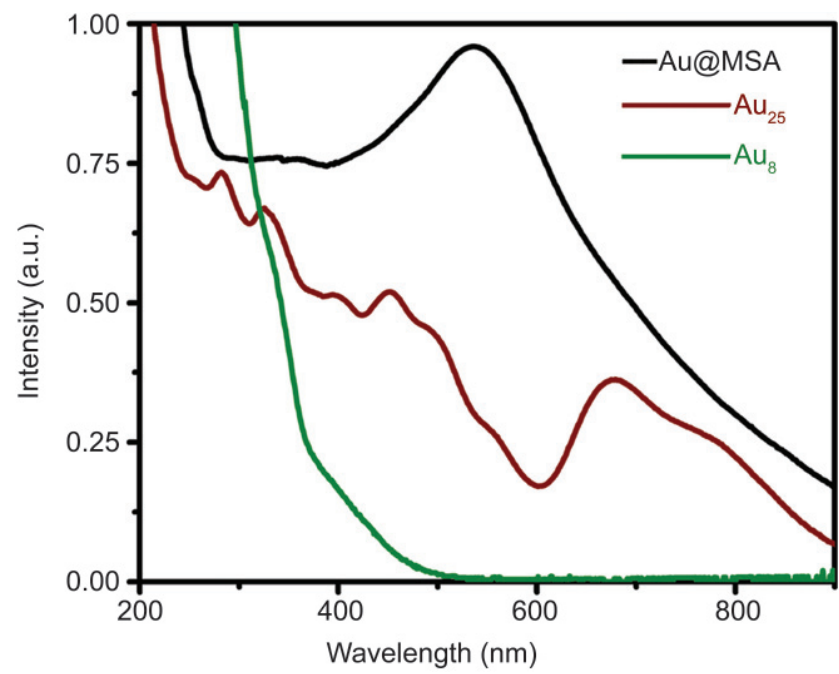

Figure 1 Comparison of the optical absorption profiles of Au@MSA, $\mathrm{Au}_{25}$ and $\mathrm{Au}_{8}$. The experimentally obtained spectral data, $I(w)$, which are functions of wavelength, were converted to the energy dependent data, $I(E)$, according to the following relation, $I(E)=I(w) /(\partial E / \partial w)$ $\alpha I(w) \times w^{2}$ where $\partial E / \partial w$ represents the Jacobian factor 
electrospray ionization mass spectrometry (ESI-MS). The supernatant obtained after etching at $\mathrm{pH} 8$ was analysed and showed a dominant peak at $\mathrm{m} / \mathrm{z} 1667$ (Fig. 2). This peak can be assigned as $\left[\mathrm{Au}_{8} \cdot 5 \mathrm{H}_{2} \mathrm{O}\right] \mathrm{H}^{+}$. An adduct of this species with a dendrimer has previously been observed when $\mathrm{Au}_{8}$ was encapsulated inside the dendrimer [7]. Other peaks with different numbers of water molecules were also seen in the spectrum. Peaks due to $\mathrm{Au}_{7}, \mathrm{Au}_{6}$, etc. fragments with different numbers of water molecules were also seen. Thus from the ESI-MS analysis, it is confirmed that the product obtained after etching at $\mathrm{pH} 8$ is $\mathrm{Au}_{8}$. The precise nature of the protection of the core by the ligand is unclear from the mass spectrum but it is likely that it is covered with -SG groups.

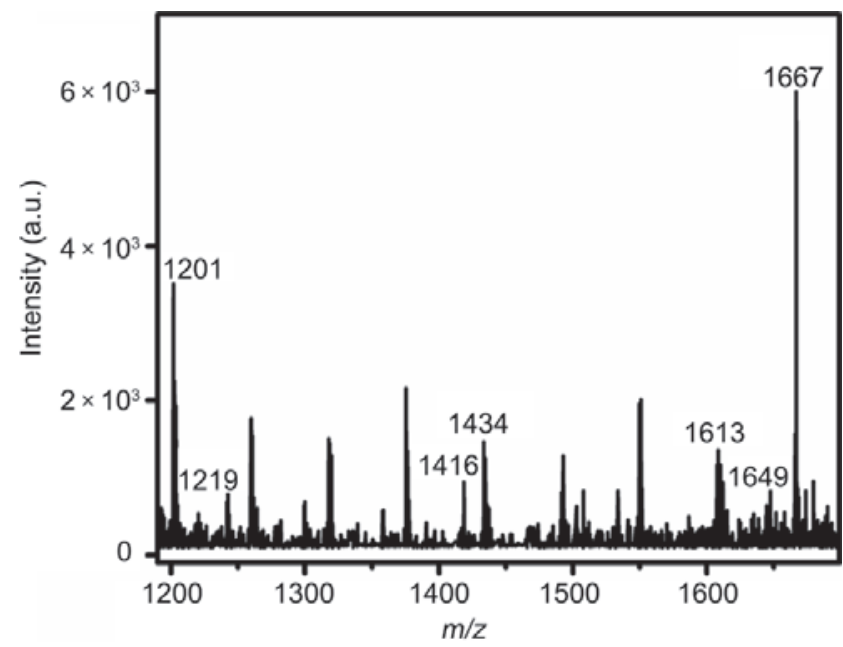

Figure 2 ESI-MS (positive mode) of $\mathrm{Au}_{8}$ in 1:1 water-methanol mixture. The peaks at $\mathrm{m} / \mathrm{z} 1667,1649,1613,1434,1416,1219$ and 1201 are due to $\left[\mathrm{Au}_{8} \cdot 5 \mathrm{H}_{2} \mathrm{O}\right] \mathrm{H}^{+},\left[\mathrm{Au}_{8} \cdot 4 \mathrm{H}_{2} \mathrm{O}\right] \mathrm{H}^{+},\left[\mathrm{Au}_{8} \cdot 2 \mathrm{H}_{2} \mathrm{O}\right] \mathrm{H}^{+}$, $\left[\mathrm{Au}_{7} \cdot 3 \mathrm{H}_{2} \mathrm{O}\right] \mathrm{H}^{+},\left[\mathrm{Au}_{7} \cdot 2 \mathrm{H}_{2} \mathrm{O}\right] \mathrm{H}^{+},\left[\mathrm{Au}_{6} \cdot 2 \mathrm{H}_{2} \mathrm{O}\right] \mathrm{H}^{+}$and $\left[\mathrm{Au}_{6} \cdot \mathrm{H}_{2} \mathrm{O}\right] \mathrm{H}^{+}$, respectively

The products after etching were analyzed using high resolution transmission electron microscopy (HRTEM) to confirm the formation of sub-nanometer sized molecular clusters. The as-prepared Au@ MSA nanoparticles have an average diameter of $4^{-}$ $5 \mathrm{~nm}$ (Fig. 3(a)). In contrast, the sub-nanometer $\mathrm{Au}_{25}$ clusters are only barely seen in the transmission electron microscopy (TEM) image (Fig. 3(b)) and no particles of larger size were observed, indicating that they have reacted completely, in agreement with the absorption spectra. Individual $\mathrm{Au}_{25}$ clusters can be seen and are marked with circles. A higher concentration of $\mathrm{Au}_{25}$ on the TEM grid gave larger

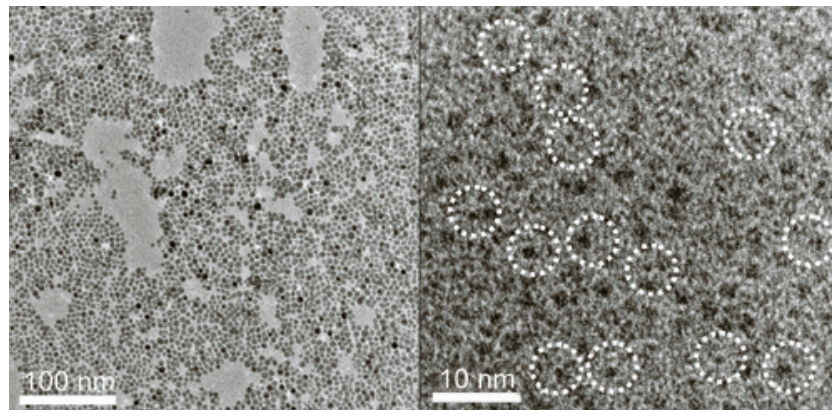

(a)

(b)

Figure 3 TEM images of (a) Au@MSA nanoparticles and (b) $\mathrm{Au}_{25}$. The clusters in (b) are seen only faintly since the size is $\sim 1 \mathrm{~nm}$. Some of the individual clusters are shown by circles. There are also cluster aggregates which upon extended electron beam irradiation fuse to form bigger particles

particles upon longer electron beam irradiation of the sample, due to core fusion as is typical of clusters in this size range. This aspect was separately checked with authentic $\mathrm{Au}_{25} \mathrm{SG}_{18}$ clusters, prepared separately using polyacrylamide gel electrophoresis (PAGE) [19]. On the other hand, nothing was seen on the grid when a solution of $\mathrm{Au}_{8}$ was spotted.

The starting material Au@MSA is not fluorescent, as it is metallic. However, both $\mathrm{Au}_{25}$ and $\mathrm{Au}_{8}$ are too small to possess a continuous density of states but rather show quantum confined electronic transitions and thus are fluorescent. Figure 4 depicts the photoluminescence profiles of $\mathrm{Au} @ \mathrm{MSA}, \mathrm{Au}_{25}$ and $\mathrm{Au}_{8} . \mathrm{Au}_{8}$ showed strong bluish green luminescence (inset of Fig. 4) with excitation and emission maxima at 370 and $465 \mathrm{~nm}$, respectively. The fluorescence from $\mathrm{Au}_{8}$ was imaged by epifluorescence microscopy (Fig. S-2 in the ESM). Au ${ }_{8}$ dissolved in ultra pure water was drop cast on a glass slide, dried, covered with a cover slip and illuminated at a wavelength of 330-380 nm. A strong bluish green fluorescence was seen from the various islands formed by the $\mathrm{Au}_{8}$ clusters. On the other hand, $\mathrm{Au}_{25}$ was more weakly fluorescent than $\mathrm{Au}_{8}$. In the photoluminescence spectrum, $\mathrm{Au}_{25}$ showed an excitation maximum at $535 \mathrm{~nm}$ and an emission maximum at $700 \mathrm{~nm}$. The excitation profile of $\mathrm{Au}_{25}$ is different from its absorption profile. The peak at $672 \mathrm{~nm}$, observed in the absorption spectrum, is not present in the excitation profile as reported earlier [19]. The excitation spectrum of $\mathrm{Au}_{25}$ showed some differences in the 300 to $600 \mathrm{~nm}$ region compared to that 


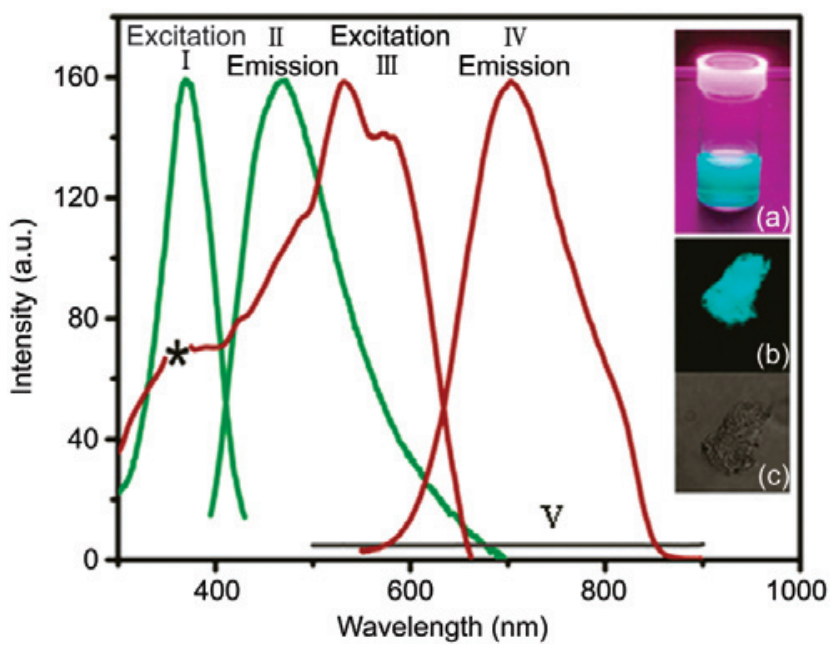

Figure 4 Comparison of the photoluminescence profiles of the clusters with Au@MSA. Traces I and II are the excitation and emission spectra of $\mathrm{Au}_{8}$, respectively. Traces III and IV are the excitation and emission spectra of $\mathrm{Au}_{25}$, respectively, and trace $\mathrm{V}$ is the emission spectrum of Au@MSA. Insets: (a) photograph showing bluish green fluorescence from $\mathrm{Au}_{8}$ in water when irradiated with a UV lamp. (b) and (c) confocal fluorescence image and corresponding bright field image, respectively, of $\mathrm{Au}_{8}$ in the solid state

previously reported [19] and therefore spectra were recorded on various instruments. Minor differences were found between spectra, attributed to detector efficiencies [23, 24]. The inherent fluorescent image of $\mathrm{Au}_{25}$ recorded by a confocal Raman spectrometer equipped with an argon ion laser (excitation wavelength $514.5 \mathrm{~nm}$ ) showed strong fluorescence from the $\mathrm{Au}_{25}$-rich region (Fig. S-3 in the ESM). $\mathrm{Au}_{25}$ showed solid state emission at $700 \mathrm{~nm}$, which is the fluorescence maximum. Since both $\mathrm{Au}_{25}$ and $\mathrm{Au}_{8}$ exhibit fluorescence, they can be used for diagnostic applications as they are highly biocompatible due to their low metallic content. The luminescence from the clusters may be due to the inter-band transitions between the filled $5 \mathrm{~d}^{10}$ band and $6(\mathrm{sp})^{1}$ conduction band [28]. The quantum yields calculated for $\mathrm{Au}_{8}$ and $\mathrm{Au}_{25}$ were 0.15 and $1.9 \times 10^{-3}$, respectively, which are in agreement with the reported values $[9,19]$. Fluorescence decay of $\mathrm{Au}_{8}$ in water was measured using two facilities. The data obtained using a picosecond resolved time correlated single photon counting (TCSPC) technique (instrument response function, $I R F=70 \mathrm{ps}$ ) are shown in the inset of Fig. 5. The numerical fitting of the fluorescence at 445 $\mathrm{nm}$ revealed time constants of 30 ps (53\%), 310 ps (22\%), $1.6 \mathrm{~ns}(18 \%)$, and $5.3 \mathrm{~ns}(7 \%)$. The existence of a significant contribution from the 30 ps component, which is comparable to the IRF of our TCSPC system (70 ps) demands the exploration of the fluorescence dynamics with much better time resolution. For this we used a femtosecond resolved fluorescence upconversion technique (IRF=165 fs) and confirmed the existence of two more time components (Fig. 5) of 0.4 ps and $6.3 \mathrm{ps}$, with equal contribution, along with the 30 ps component as revealed by the TCSPC technique. The lifetime values obtained here are different from those reported for $\mathrm{Au}_{8}$, synthesized inside the dendrimer [7]. $\mathrm{Au}_{8}$ encapsulated inside the dendrimer showed two lifetime components, $7.5 \mathrm{~ns}(93 \%)$ and $2.8 \mu \mathrm{s}(7 \%)$ which are due to singlet transitions between low-lying $\mathrm{d}$ bands and excited sp bands and a triplet-singlet intraband transition, respectively [7]. The difference in the lifetime values can be attributed to two factors. Firstly, in our case $\mathrm{Au}_{8}$ is free in solution and not encapsulated inside a dendrimer. As a result, the degree of freedom is more than the encapsulated $\mathrm{Au}_{8}$. Secondly, in the reported lifetime measurements, the IRF is about 7-8 ns and the shorter lifetime reported is $7.5 \mathrm{~ns}$. Lifetime values below 7.5 ns cannot be measured with this resolution. On the other hand, we have an IRF of $165 \mathrm{fs}$ in our femtoseond system which allows the measurement of short lifetimes. The dynamics of $\mathrm{Au}_{25}$ emission were

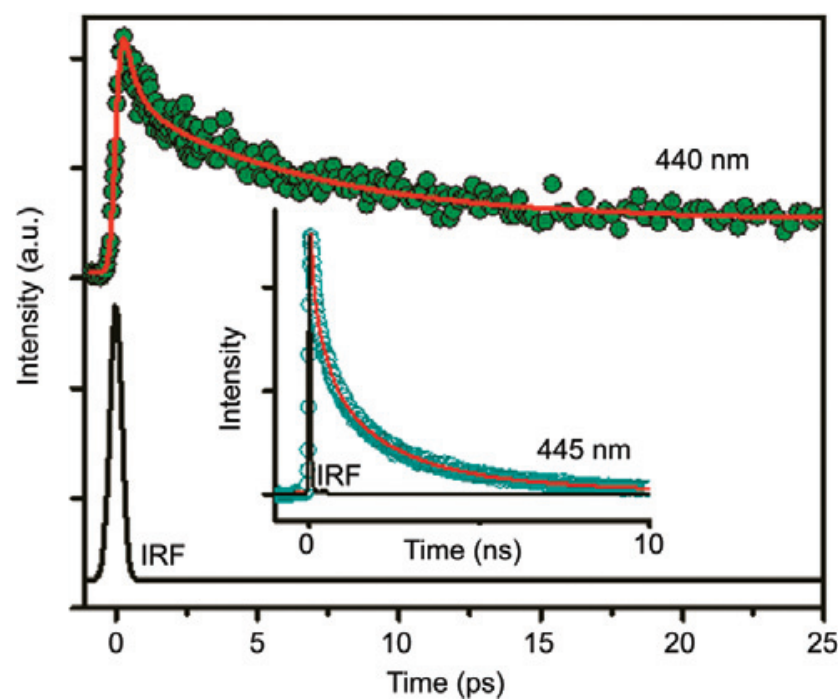

Figure 5 Fluorescence decay profile of $\mathrm{Au}_{8}$ in water at $440 \mathrm{~nm}$ (excitation at $364 \mathrm{~nm}$ ) obtained by the fluorescence up-conversion technique (IRF=165 fs). Baseline of the transient is shifted for clarity. Inset shows the fluorescence transient of the sample at $445 \mathrm{~nm}$ (excitation at $375 \mathrm{~nm})$ in the longer time window of a TCSPC (IRF=70 ps) set-up 
also studied, and matched the dynamics of authentic $\mathrm{Au}_{25} \mathrm{SG}_{18}$ prepared by electrophoresis [19].

The next step was to check whether the $\mathrm{Au}_{25}$ cluster is completely protected with glutathione, so that it can be correctly denoted $\mathrm{Au}_{25} \mathrm{SG}_{18}$. There is a probability that some MSA molecules are present. To confirm the presence of glutathione on the cluster surface, a ${ }^{1} \mathrm{H}$ NMR spectrum was recorded (Fig. S-4 in the ESM). The NMR spectrum of $\mathrm{Au}_{25}$ showed features due to glutathione. The peaks were broadened and shifted slightly to higher ppm values when compared with free GSH due to the non-uniform distribution of ligands on the metal surface and the dipolar spin relaxation of the ligand on the densely packed region near the thiolate/gold interface. Free GSH in $\mathrm{D}_{2} \mathrm{O}$ showed five characteristic resonances [22-24] as shown in trace II, which can be assigned as indicated in Fig. S-4. The methylene close to $-\mathrm{SH}$ of the cysteine residue of glutathione, labeled as ' $c$ ' in Fig. S-4, broadened to the baseline when it was bound on the $\mathrm{Au}_{25}$ surface (trace IV) [22 -24]. This absence is as expected when thiolate is bound to the $\mathrm{Au}_{25}$ core. However, no MSA features were manifested in the spectrum of $\mathrm{Au}_{25}$. Fourier transform infrared (FT-IR) spectra were measured to further confirm the presence of glutathione on the $\mathrm{Au}_{25}$ surface. The FT-IR spectra of MSA, Au@MSA, GSH and $\mathrm{Au}_{25}$ are compared in Fig. S-5 (in the ESM). The spectrum of $\mathrm{Au}_{25}$ showed (trace d) characteristic features of glutathione. The loss of the S-H stretching vibration at $2526 \mathrm{~cm}^{-1}$ when compared with free glutathione (trace c) confirms that $\mathrm{Au}_{25}$ is covered with -SG groups. ESIMS of $\mathrm{Au}_{25}$ formed after etching (Fig. S-6 in the ESM) showed peaks due to -SG, AuSG, $\mathrm{AuSG}_{2}, \mathrm{Au}_{2} \mathrm{SG}_{2}$, etc. No MSA features were observed. This confirms the presence of glutathione and absence of MSA on the cluster surface. ESI-MS in the orthogonal geometry such ours does not give features due to intact $\mathrm{Au}_{25} \mathrm{SG}_{18}$, as reported previously [19]. Thus the NMR, FT-IR, and ESI-MS confirm complete -SG protection of the cluster and we can confidently assign the product as $\mathrm{Au}_{25} \mathrm{SG}_{18}$. As the $\mathrm{Au}_{8}$ obtained even after precipitation and purification cycles contained free glutathione which could not be removed completely, FT-IR and NMR measurements showed features due to free glutathione. The nature of ligand protection of $\mathrm{Au}_{8}$ is unclear at this time. Efforts are underway to purify the cluster and to obtain a clean ESI-MS spectrum in order to assign the ligand structure.

The mechanism of formation of the clusters is not well understood. We present below our tentative suggestions for the formation of $\mathrm{Au}_{25}$. There are two possible routes for etching (Fig. 6). In the first route, gold atoms are removed from the surface of the nanoparticle by excess glutathione as gold(I) glutathione thiolate. The gold(I) glutathione complex may undergo strong aurophilic interactions-as is the tendency for gold(I) compounds-to form dimers, oligomers, chains, or layers via gold(I)gold(I) interactions due to the hybridization of the empty $6 s / 6 p$ and filled $5 d$ orbitals to form $\mathrm{Au}_{25} \mathrm{SG}_{18}$, as this is the most stable cluster among the glutathione protected gold clusters in the lower size range. In the second possible route, glutathione may etch the surface gold atoms of Au@MSA leading to a reduction in size of the nanoparticles in steps, resulting in the formation of $\mathrm{Au}_{25} \mathrm{SG}_{18}$. The gold atoms removed from the nanoparticle surface by glutathione deposit as gold(I) glutathione thiolate which was obtained as a precipitate after etching. The same explanation can also be given for $\mathrm{Au}_{8}$. Recently, it was reported that gold nanoparticle

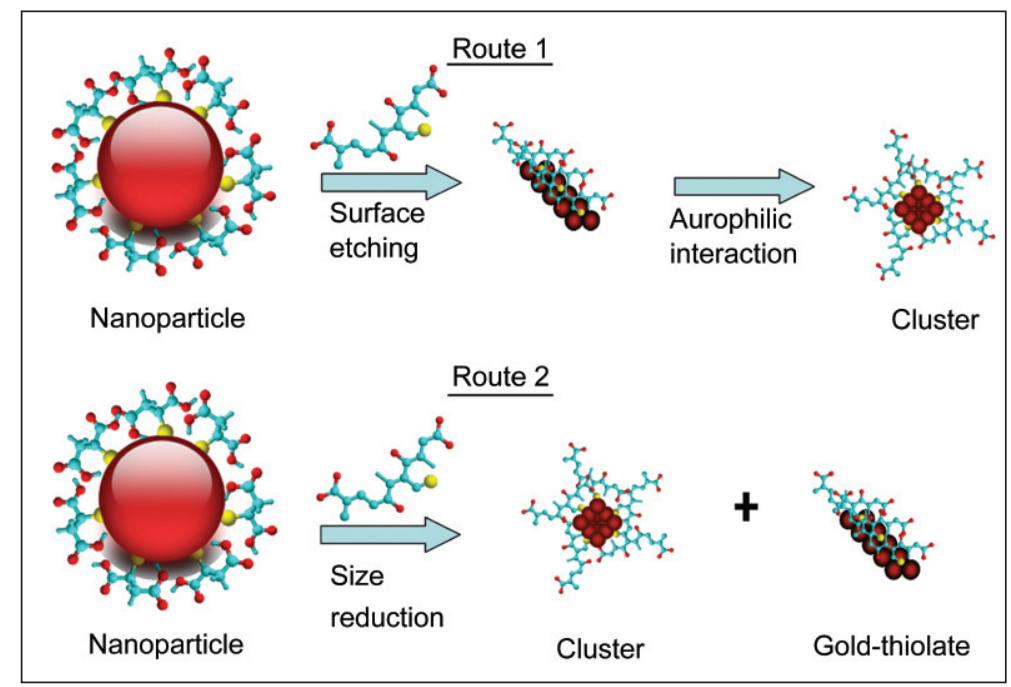

Figure 6 Schematic representation of two possible routes for the formation of clusters from Au@MSA nanoparticles 
size can be controlled by the size of the polymeric $\mathrm{Au}(\mathrm{I})$ thiolate precursor [29]. It was suggested that lower $\mathrm{pH}$ values favor larger and denser polymeric precursors and higher $\mathrm{pH}$ values favor smaller and less dense precursors. Consequently, the larger precursors lead to the formation of larger Au clusters, whereas smaller precursors lead to the formation of smaller $\mathrm{Au}$ clusters. Assuming that $\mathrm{Au}(\mathrm{I})$ thiolate is formed as an intermediate during etching, reaction at lower $\mathrm{pH}$ should lead to the formation of larger $\mathrm{Au}_{25}$ clusters and that at higher $\mathrm{pH}$ should give smaller $\mathrm{Au}_{8}$ clusters.

In conclusion, we have synthesized two magic number clusters, $\mathrm{Au}_{25}$ and $\mathrm{Au}_{8}$, from $\mathrm{Au} @ \mathrm{MSA}$ nanoparticles via ligand etching with glutathione. The two clusters are highly fluorescent and can be viable options for in vivo studies since they are highly biocompatible due to their low metallic content. Due to the possibility of various ligand functionalizations $[23,24]$ and the possibility of obtaining fluorescence resonance energy transfer in such variously functionalized $\mathrm{Au}_{25}$ [24], there are numerous applications in biology which demands a fast and efficient synthesis such as that reported here.

\section{Experimental}

Chemicals: The following reagents were used as received. Chloroauric acid trihydrate $\left(\mathrm{HAuCl}_{4} \cdot 3 \mathrm{H}_{2} \mathrm{O}\right.$, 99\%) was received from CDH (P) Ltd., India. Mercaptosuccinic acid, glutathione and sodium borohydride were received from Sigma Aldrich. Methanol (99.8\%) and ethanol (99.5\%) of reagent grade were received from Merck Chemical Co.

Synthesis of MSA-protected gold nanoparticles (Au@MSA): Water soluble, metallic MSA-protected gold nanoparticles were prepared by a method similar to the one reported earlier [30, 31]. To 100 $\mathrm{mL}$ of $5 \mathrm{mmol} / \mathrm{L} \mathrm{HAuCl}_{4}$ in methanol, $10 \mathrm{mmol} /$ $\mathrm{L}$ mercaptosuccinic acid (MSA) was added. The mixture was then cooled to $0{ }^{\circ} \mathrm{C}$ in an ice bath for $30 \mathrm{~min}$. An aqueous solution of $\mathrm{NaBH}_{4}(25 \mathrm{~mL}, 0.2$ $\mathrm{mol} / \mathrm{L}$ ), cooled at $0{ }^{\circ} \mathrm{C}$, was injected rapidly into this mixture under vigorous stirring. The mixture was allowed to react for another hour. The resulting precipitate was collected and washed repeatedly with methanol through centrifugal precipitation and dried to obtain the Au@MSA nanoparticles as a dark brown powder. This method gives nanoparticles of 4 to $5 \mathrm{~nm}$ core size.

Synthesis of $A u_{25}$ clusters: Au@MSA in water was mixed with GSH, 1:4 by weight, in air under constant stirring. The $\mathrm{pH}$ of the solution was around 3 . The temperature of the mixture was maintained at $55^{\circ} \mathrm{C}$ $\pm 5{ }^{\circ} \mathrm{C}$ with an oil bath. The reaction was monitored by optical absorption spectroscopy. Heating was discontinued when absorption features similar to $\mathrm{Au}_{25}$ appeared in the UV-Vis spectrum. This typically took 12 hours. The solution was centrifuged and methanol was added to the supernatant to precipitate the cluster. The precipitate was dried to obtain the clusters in the powder form. The powder redissolved in water showed an absorption maximum at $667 \mathrm{~nm}$. The powder was etched again with GSH for $6^{-} 8 \mathrm{~h}$ as per the protocol described above, giving a material which showed the well-structured spectroscopic features of $\mathrm{Au}_{25}$. The solution was centrifuged again and methanol was added to the supernatant to precipitate the cluster. The precipitate was dried to obtain a reddish brown powder. The resulting powder was redissolved in water and the UV-Vis spectrum showed peaks characteristic of $\mathrm{Au}_{25}$ which has a well-structured optical absorption spectrum with a characteristic absorption maximum at $672 \mathrm{~nm}$ with absorption intensity increasing towards the lower wavelength region. From the amounts used in the synthesis, we estimate that the $\mathrm{Au}_{25}$ yield is $55 \% \pm 5 \%$.

Synthesis of $A u_{8}$ clusters: Au@MSA in water was mixed with GSH, 1:4 by weight, in air under constant stirring. The $\mathrm{pH}$ of the solution was adjusted to 8 by adding $\mathrm{NaOH}$. The temperature of the mixture was maintained at $60{ }^{\circ} \mathrm{C} \pm 5{ }^{\circ} \mathrm{C}$ with an oil bath. After 15-20 hours, the solution was centrifuged and ethanol was added to the supernatant to precipitate the cluster. The precipitate was dried to obtain the clusters in the powder form. Due to the presence of excess glutathione in the product, the yield of $\mathrm{Au}_{8}$ could not be estimated.

Details of instrumentation: UV/Vis spectra were measured with a Perkin Elmer Lambda 25 instrument in the range $200-1100 \mathrm{~nm}$. Fourier transform infrared (FT-IR) spectra were measured with a Perkin Elmer 
Spectrum One instrument. KBr crystals were used as the matrix for preparing samples. High resolution transmission electron microscopy of clusters was carried out with a JEOL 3010 instrument. The microscope was operated at $200 \mathrm{keV}$ to reduce beaminduced damage of the clusters. Nanoparticles were measured at $300 \mathrm{keV}$. The samples were drop cast on carbon-coated copper grids and allowed to dry under ambient conditions. The Fourier transform nuclear magnetic resonance (FT-NMR) measurements were recorded with a JEOL $500 \mathrm{MHz}$ instrument. The solvent used was $\mathrm{D}_{2} \mathrm{O}$. The electrospray ionization (ESI) mass spectrometric measurements were obtained with an MDX Sciex 3200 Q TRAP LC। MS $\backslash$ MS instrument in which the spray and the extraction are orthogonal to each other. The samples taken in 1:1 water-methanol were electrosprayed at $5 \mathrm{kV}$. The spectra were averaged for 100 scans. Fluorescent measurements were carried out on Cary Eclipse instrument. The band pass for excitation and emission was set as $5 \mathrm{~nm}$. A WITec $\mathrm{GmbH}$ confocal Raman spectrometer, equipped with an argon ion laser $(514.5 \mathrm{~nm})$ was used as the excitation source to collect the fluorescence images. The laser was focused onto the sample using a 100x objective with the signal collected in a back scattering geometry. The signal, after passing through a super notch filter, was dispersed using a 150 grooves/mm grating onto a Peltier-cooled charge coupled device (CCD), which served as the detector. The sample mounted on a piezo stage was scanned with signals collected at every pixel. For the images displayed, the scan area was divided into $100 \times 100$ pixels for spectral image acquisition. The TCSPC setup was from Edinburgh Instruments (LifeSpec-ps) with PicoQuant laser diode excitation $(375 \mathrm{~nm})$. The emission decay at $445 \mathrm{~nm}$ was fitted by the software supplied with the instrument. In our femtosecond up-conversion setup (FOG 100, CDP), the sample was excited at 364 $\mathrm{nm}$ using the second harmonic of a mode-locked Tisapphire laser (Tsunami, Spectra Physics) pumped by a $10 \mathrm{~W}$ Millennia laser (Spectra Physics) and emission was observed at $440 \mathrm{~nm}$. The femtosecond fluorescence decays were fitted using SCIENTIST ${ }^{\mathrm{TM}}$ software. The experimentally obtained intensities in absorbance, as a function of wavelength, $I(w)$ were converted to energy dependent values, $I(E)$ using the expression, $I(E)=I(w) /(\partial E / \partial w) \alpha I(w) \times w^{2}$ where $\partial E / \partial w$ represents the Jacobian factor.

\section{Acknowledgments}

We thank Department of Science and Technology, Government of India for constantly supporting our research program on nanomaterials.

Electronic Supplementary Material: Supplementary material is available in the online version of this article at http://dx.doi.org/10.1007/s12274-008-8035-2 and is accessible free of charge.

\section{References}

[1] Balamurugan, B.; Maruyama, T. Evidence of an enhanced interband absorption in Au nanoparticles: Sizedependent electronic structure and optical properties. Appl. Phys. Lett. 2005, 87, 143105.

[2] Jain, P. K.; Huang, X.; El-Sayed, I. H.; El-Sayed, M. A. Noble metals on the nanoscale: Optical and photothermal properties and some applications in imaging, sensing, biology, and medicine. Acc. Chem. Res. 2008, in press, DOI: 10.1021/ar7002804.

[3] Guo, R.; Song, Y.; Wang, G.; Murray, R. W. Does core size matter in the kinetics of ligand exchanges of monolayerprotected Au clusters? J. Am. Chem. Soc. 2005, 127, 2752-2757.

[4] Duan, C.; Cui, H.; Zhang, Z.; Liu, B.; Guo, J.; Wang, W. Size-dependent inhibition and enhancement by gold nanoparticles of luminol-ferricyanide chemiluminescence. J. Phys. Chem. C 2007, 111, 4561-4566.

[5] Ramakrishna, G.; Ghosh, H. N. Effect of particle size on the reactivity of quantum size $\mathrm{ZnO}$ nanoparticles and charge-transfer dynamics with adsorbed catechols. Langmuir 2003, 19, 3006-3012.

[6] Zheng, J.; Nicovich, P. R.; Dickson, R. M. Highly fluorescent noble-metal quantum dots. Annu. Rev. Phys. Chem. 2007, 58, 409-431.

[7] Zheng, J.; Petty, J. T.; Dickson, R. M. High quantum yield blue emission from water-soluble $\mathrm{Au}_{8}$ nanodots. J. Am. Chem. Soc. 2003, 125, 7780-7781.

[8] Zheng, J.; Zhang, C. W.; Dickson, R. M. Highly fluorescent, water-soluble, size-tunable gold quantum dots. Phys. Rev. Lett. 2004, 93, 077402.

\section{国备 Springer}


[9] Duan, H.; Nie, S. Etching colloidal gold nanocrystals with hyperbranched and multivalent polymers: A new route to fluorescent and water-soluble atomic clusters. J Am. Chem. Soc. 2007, 129, 2412-2413.

[10] Woehrle, G. H.; Hutchison, J. E. Thiol-functionalized undecagold clusters by ligand exchange: Synthesis, mechanism, and properties. Inorg. Chem. 2005, 44, 6149-6158.

[11] Bertino, M. F.; Sun, Z.-M.; Zhang, R.; Wang, L.-S. Facile syntheses of monodisperse ultrasmall Au clusters. J. Phys. Chem. B 2006, 110, 21416-21418.

[12] Nunokawa, K.; Onaka, S.; Ito, M.; Horibe, M.; Yonezawa, T.; Nishihara, H.; Ozeki, T.; Chiba, H.; Watase, S.; Nakamoto, M. Synthesis, single crystal X-ray analysis, and TEM for a single-sized $\mathrm{Au}_{11}$ cluster stabilized by SR ligands: The interface between molecules and particles. J. Organomet. Chem. 2006, 691, 638-642.

[13] Yanagimoto, Y.; Negishi, Y.; Fujihara, H.; Tsukuda, T. Chiroptical activity of BINAP-stabilized undecagold clusters. J. Phys. Chem. B 2006, 110, 11611-11614.

[14] Woehrle, G. H.; Warner, M. G.; Hutchison, J. E. Ligand exchange reactions yield subnanometer, thiol-stabilized gold particles with defined optical transitions. J. Phys. Chem. B 2002, 106, 9979-9981.

[15] Menard, L. D.; Gao, S.-P.; Xu, H.; Twesten, R. D.; Harper, A. S.; Song, Y.; Wang, G.; Douglas, A. D.; Yang, J. C.; Frenkel, A. I. et al. Sub-nanometer Au monolayer-protected clusters exhibiting molecule-like electronic behavior: Quantitative high-angle annular dark-field scanning transmission electron microscopy and electrochemical characterization of clusters with precise atomic stoichiometry. J. Phys. Chem. B 2006, 110, 12874-12883.

[16] Abad, J. M.; Sendroiu, I. E.; Gass, M.; Bleloch, A.; Mills, A. J.; Schiffrin D. J. Synthesis of $\omega$-hydroxy hexathiolateprotected subnanometric gold clusters. J. Am. Chem. Soc. 2007, 129, 12932-12933.

[17] Menard, L. D.; Xu, H.; Gao, S. P.; Twesten, R. D.; Harper, A. S.; Song, Y.; Wang, G.; Douglas, A. D.; Yang, J. C.; Frenkel, A. I.; et al. Metal core bonding motifs of monodisperse icosahedral $\mathrm{Au}_{13}$ and larger Au monolayerprotected clusters as revealed by X-ray absorption spectroscopy and transmission electron microscopy. J. Phys. Chem. B 2006, 110, 14564-14573.

[18] Schaaff, T. G.; Knight, G.; Shafigullin, M. N.; Borkman, R. F.; Whetten, R. L. Isolation and selected properties of a 10.4 kDa gold: Glutathione cluster compound. J. Phys. Chem. B 1998, 102, 10643-10646.
[19] Negishi, Y.; Nobusada, K.; Tsukuda, T. Glutathioneprotected gold clusters revisited: Bridging the gap between gold(I)-thiolate complexes and thiolateprotected gold nanocrystals. J. Am. Chem. Soc. 2005, 127, 5261-5270.

[20] Shichibu, Y.; Negishi, Y.; Tsukuda, T.; Teranishi, T. Largescale synthesis of thiolated $\mathrm{Au}_{25}$ clusters via ligand exchange reactions of phosphine-stabilized $\mathrm{Au}_{11}$ clusters. J. Am. Chem. Soc. 2005, 127, 13464-13465.

[21] Shichibu, Y.; Negishi, Y.; Tsunoyama, H.; Kanehara, M.; Teranishi, T.; Tsukuda T. Extremely high stability of glutathionate-protected $\mathrm{Au}_{25}$ clusters against core etching. Small 2007, 3, 835-839.

[22] Habeeb Muhammed, M. A.; Pradeep, T. Reactivity of $\mathrm{Au}_{25}$ clusters with $\mathrm{Au}^{3+}$. Chem. Phys. Lett. 2007, 449, 186-190.

[23] Shibu, E. S.; Habeeb Muhammed, M. A.; Tsukuda, T.; Pradeep, T. Ligand exchange of $\mathrm{Au}_{25} \mathrm{SG}_{18}$ leading to functionalized gold clusters: spectroscopy, kinetics, and luminescence. J. Phys. Chem C 2008, 112, 12168-12176.

[24] Habeeb Muhammed, M. A.; Shaw, A. K.; Pal, S. K.; Pradeep T. Quantum clusters of gold exhibiting FRET. J. Phys. Chem C, 2008, 112, 14324-14330.

[25] Zhu, M.; Lanni, E.; Garg, N.; Bier, M. E.; Jin, R. Kinetically controlled, high-yield synthesis of $\mathrm{Au}_{25}$ clusters. J. Am. Chem. Soc. 2008, 130, 1138-1139.

[26] Lee, T.-H.; Gonzalez, J. I.; Zheng, J.; Dickson, R. M. Single-molecule optoelectronics. Acc. Chem. Res. 2005, 38, 534-541.

[27] Zhu, M.; Aikens, C. M.; Hollander, F. J.; Schatz, G. C.; Jin, R. Correlating the crystal structure of a thiol-protected $\mathrm{Au}_{25}$ cluster and optical properties. J. Am. Chem. Soc. 2008, 130, 5883-5885.

[28] Huang, T.; Murray, R. W. Visible luminescence of watersoluble monolayer-protected gold clusters. J. Phys. Chem. B 2001, 105, 12498-12502.

[29] Brinas, R. P.; Hu, M.; Qian, L.; Lymar, E. S.; Hainfeld, J. F. Gold nanoparticle size controlled by polymeric $\mathrm{Au}(\mathrm{I})$ thiolate precursor size. J. Am. Chem. Soc. 2008, 130, 975-982.

[30] Nishida, N.; Shibu, E. S.; Yao, H.; Oonishi, T.; Kimura, K.; Pradeep, T. Fluorescent gold nanoparticle superlattices. Adv. Mater. 2008, in press, DOI: 10.1002/ adma.200800632.

[31] Chen, S.; Kimura, K. Synthesis and characterization of carboxylate-modified gold nanoparticle powders dispersible in water. Langmuir 1999, 15, 1075-1082. 